\title{
A Hybrid Time-Domain Discontinuous Galerkin-Boundary Integral Method for Electromagnetic Scattering Analysis
} للعلوم والتقنية King Abdullah University of Science and Technology

\section{Ping $\mathrm{Li}^{\dagger}$, Yifei Shi}

$\dagger$ The University of Hong Kong, Hong Kong, China

‡ King Abdullah University of Science and Technology (KAUST), Saudi Arabia

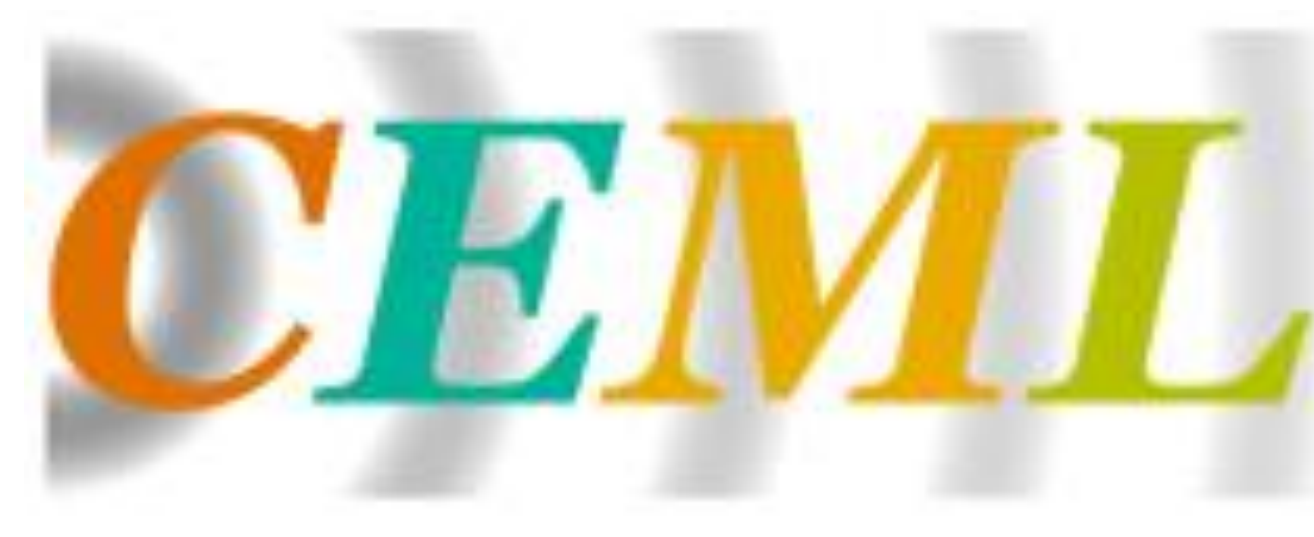

PROBLEM

A hybrid discontinuous Galerkin time domain (DGTD)-time domain boundary integral (TDBI) method is proposed for analyzing transient scattering from penetrable scatterers. The radiation condition is rigorously enforced via the incoming numerical flux on the truncation domain boundary of the computation domain. The fields required by flux are computed using TDBI method from equivalent currents introduced on a Huygens' surface enclosing the scatters.

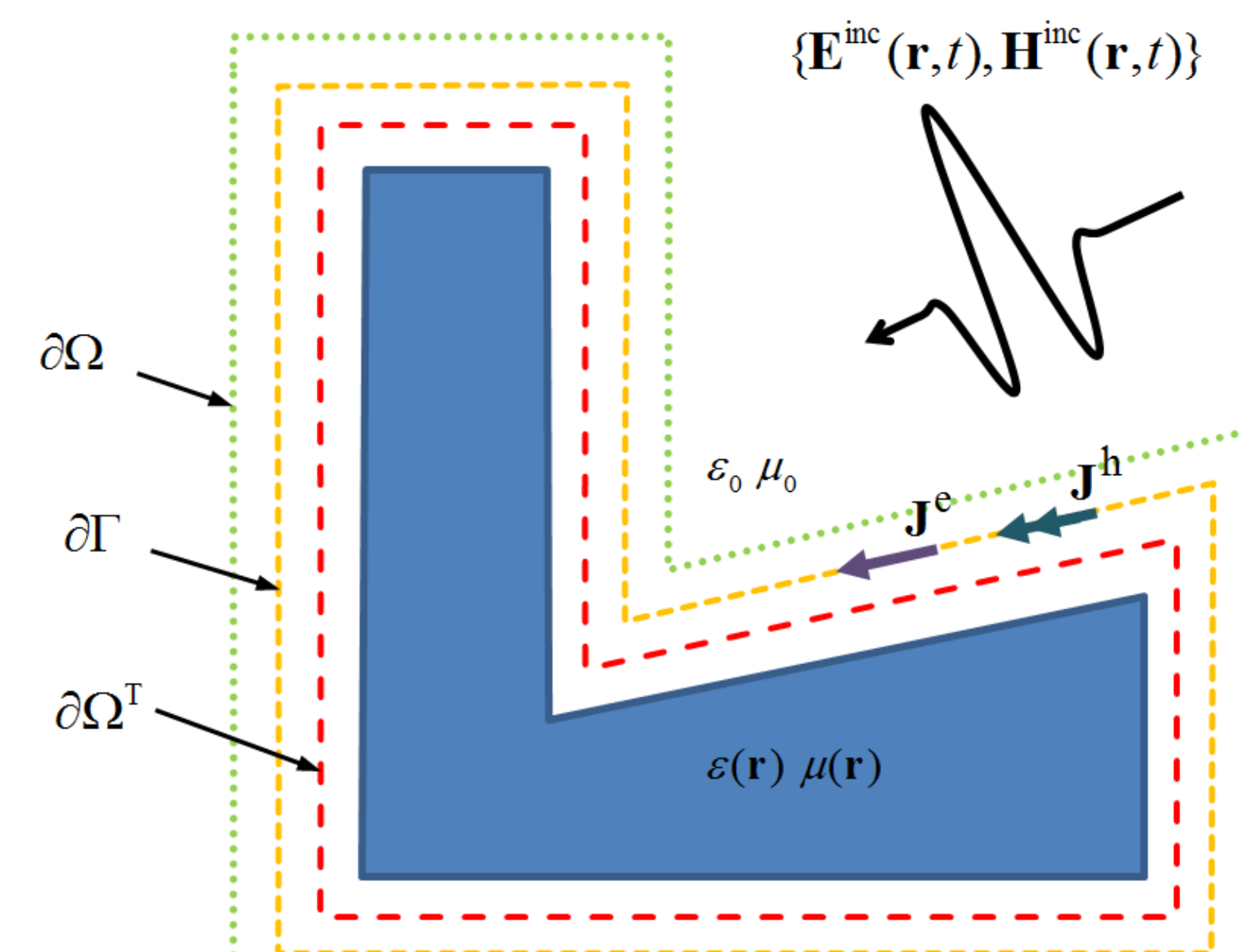

(r) Permittivity Normal unit vector $\hat{\mathbf{n}}$

(r) Permeability $\quad$ Position vector $\mathbf{r}$

$c$ Speed of light $\quad$ Distance $R$

J Electric current Surface $S$

$\mathbf{E}^{\text {inc }}$ Incident electric field $\backslash$ Time $t$

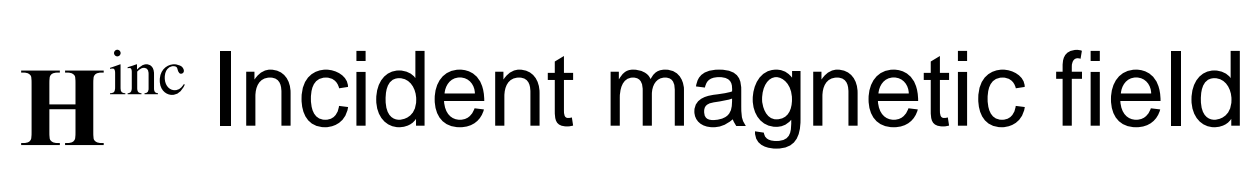

$\varepsilon_{0} \quad$ Free space permittivity

$\mu_{0} \quad$ Free space permeability

DGTD Formulation

In each tetrahedral elements $\Omega_{i}$ [1], fields are represented as

$$
\mathbf{E}_{i}(\mathbf{r}, t)={ }_{k=1}^{d_{i}^{\mathrm{e}}} f_{i, k}^{\mathrm{e}}(t) \mathbf{v}_{i, k}^{\mathrm{e}}(\mathbf{r}) \quad \mathbf{H}_{i}(\mathbf{r}, t)={ }_{i=1}^{d_{i}^{\mathrm{h}}} f_{i, h}^{\mathrm{h}}(t) \mathbf{v}_{i, l}^{\mathrm{h}}(\mathbf{r})
$$

The Maxwell curl equations are tested by edge vector basis functions $\mathbf{v}_{i, l}^{\mathrm{h}}$ and $\mathbf{v}_{i, r^{*}}^{\mathrm{e}}$

$$
\begin{aligned}
& \int \mathbf{v}_{i, k}^{\mathrm{e}} \cdot\left[\begin{array}{ll}
{ }_{i} \partial_{t} \mathbf{E}_{i} & \nabla \times \mathbf{H}_{i}
\end{array}\right] d \mathbf{r}=\sum_{m=1}^{4} \int_{\partial} \mathbf{v}_{i, k}^{\mathrm{e}} \cdot\left[\begin{array}{ll}
\hat{\mathbf{n}}_{i, m} \times\left(\mathbf{H}_{m}\right. & \left.\mathbf{H}_{i}\right)
\end{array}\right] d \mathbf{r} \\
& \int_{i, l}^{i} \mathbf{v}_{i, l}^{\mathrm{h}} \cdot\left[{ }_{i} \partial_{t} \mathbf{H}_{i}+\nabla \times \mathbf{E}_{i}\right] d \mathbf{r}=\sum_{m=1}^{4} \int_{\partial}^{l, m} \mathbf{v}_{i, l}^{\mathrm{h}} \cdot\left[\hat{\mathbf{n}}_{i, m} \times\left(\mathbf{E}_{i} \quad \mathbf{E}_{m}\right)\right] d \mathbf{r}
\end{aligned}
$$

The upwind numerical fluxes are

$$
\begin{aligned}
& \hat{\mathbf{n}}_{i, m} \times \mathbf{H}_{m}^{*}=\hat{\mathbf{n}}_{i, m} \times\left(Z_{i} \mathbf{H}_{i}+\hat{\mathbf{n}}_{i, m} \times \mathbf{E}_{i}+\tilde{Z} \tilde{\mathbf{H}}-\hat{\mathbf{n}}_{i, m} \times \tilde{\mathbf{E}}\right) /\left(Z_{i}+\tilde{Z}\right) \\
& \hat{\mathbf{n}}_{i, m} \times \mathbf{E}_{m}^{*}=\hat{\mathbf{n}}_{i, m} \times\left(Y_{i} \mathbf{E}_{i}-\hat{\mathbf{n}}_{i, m} \times \mathbf{H}_{i}+\tilde{Y} \tilde{\mathbf{E}}+\hat{\mathbf{n}}_{i, m} \times \tilde{\mathbf{H}}\right) /\left(Y_{i}+\tilde{Y}\right)
\end{aligned}
$$

$\tilde{\mathbf{E}}$ and $\tilde{\mathbf{H}}$ depend on the location. $\mathbf{E}_{i, m}^{\partial \Omega}$ and $\mathbf{H}_{i, m}^{\partial \Omega}$ are calculated using TDBI. Incident fields are enforced on $\partial \Omega^{\mathrm{T}}$.

$$
\begin{aligned}
& \tilde{\mathbf{E}}= \begin{cases}\mathbf{E}_{i, m}^{\partial \Omega} & \partial \Omega_{i, m} \in \partial \Omega \\
\mathbf{E}_{j} \pm \mathbf{E}_{i, m}^{\text {inc }} & \partial \Omega_{i, m} \in \partial \Omega^{\mathrm{T}} \\
\mathbf{E}_{j} & \text { else }\end{cases} \\
& \tilde{Z}=\left\{\begin{array}{lll}
Z_{0} & \partial \Omega_{i, m} \in \partial \Omega \\
Z_{j} & \text { else }
\end{array}\right. \tilde{\mathbf{H}}= \begin{cases}\mathbf{H}_{i, m}^{\partial \Omega} & \partial \Omega_{i, m} \in \partial \Omega \\
\mathbf{H}_{j} \pm \mathbf{H}_{i, m}^{\text {inc }} & \partial \Omega_{i, m} \in \partial \Omega^{\mathrm{T}} \\
\mathbf{H}_{j} & \text { else }\end{cases}
\end{aligned}
$$

\section{TDBI Formulation}

Calculate the equivalent surface currents on $\partial \Gamma$

$$
\begin{aligned}
& \mathbf{J}_{i, m}^{\mathrm{h}}(\mathbf{r}, t)={ }_{\substack{d_{i}^{\mathrm{h}} \\
l=1}}^{\mathrm{h}} f_{i, l}^{\mathrm{h}}(t) \hat{\mathbf{n}}_{i, m}(\mathbf{r}) \quad \mathbf{v}_{i, l}^{\mathrm{h}}(\mathbf{r}) \\
& \mathbf{J}_{i, m}^{\mathrm{e}}(\mathbf{r}, t)={ }_{d_{i}^{\mathrm{h}}}^{\mathrm{h}} f_{i, k}^{\mathrm{e}}(t) \hat{\mathbf{n}}_{i, m}(\mathbf{r}) \quad \mathbf{v}_{i, k}^{\mathrm{e}}(\mathbf{r})
\end{aligned}
$$

The fields on $\partial \Omega_{i^{\prime}, m^{\prime}} \in \partial \Omega$ are obtained by radiating the equivalent currents [2]:

$$
\begin{aligned}
& \mathbf{E}_{i, m}^{\partial \Omega}(\mathbf{r}, t)=\sum_{i^{\prime}} \sum_{m^{\prime}}\left[\mu_{0} \mathcal{L}_{i^{\prime}, m^{\prime}}\left(\mathbf{J}_{i^{\prime}, m^{\prime}}^{\mathrm{h}}\right)-\mathcal{K}_{i^{\prime}, m^{\prime}}\left(\mathbf{J}_{i^{\prime}, m^{\prime}}^{\mathrm{e}}\right)\right] \\
& \mathbf{H}_{i, m}^{\partial \Omega}(\mathbf{r}, t)=\sum_{i^{\prime}} \sum_{m^{\prime}}\left[\varepsilon_{0} \mathcal{L}_{i^{\prime}, m^{\prime}}\left(\mathbf{J}_{i^{\prime}, m^{\prime}}^{\mathrm{e}}\right)+\mathcal{K}_{i^{\prime}, m^{\prime}}\left(\mathbf{J}_{i^{\prime}, m^{\prime}}\right)\right] \\
& \mathcal{K}_{i^{\prime}, m^{\prime}}(\mathbf{J})=\frac{1}{4 \pi} \nabla \times \int_{\partial \Omega_{r^{\prime}, m^{\prime}}} \frac{\mathbf{J}\left(\mathbf{r}^{\prime}, t-R / c_{0}\right)}{R} d \mathbf{r}^{\prime} \\
& \mathcal{L}_{i^{\prime}, m^{\prime}}(\mathbf{J})=-\frac{1}{4 \pi} \int_{\partial \Omega_{r^{\prime}, m^{\prime}}} \frac{\partial_{t} \mathbf{J}\left(\mathbf{r}^{\prime}, t-R / c_{0}\right)}{R} d \mathbf{r}^{\prime}+\frac{c_{0}^{2}}{4 \pi} \nabla \int_{\partial \Omega_{t^{\prime}, m^{\prime}}} \int_{0}^{t-R / c_{0}} \frac{\nabla^{\prime} \cdot \mathbf{J}\left(\mathbf{r}^{\prime}, t^{\prime}\right)}{R} d t^{\prime} d \mathbf{r}^{\prime}
\end{aligned}
$$

Final System of Equations

$$
\begin{aligned}
\varepsilon_{i} \overline{\mathbf{M}}_{i}^{\mathrm{e}} \partial_{t} \mathbf{e}_{i} & =\left[\overline{\mathbf{S}}_{i}^{\mathrm{eh}}-\overline{\mathbf{F}}_{i i}^{\mathrm{eh}}\right] \mathbf{h}_{i}+\overline{\mathbf{F}}_{i i}^{\mathrm{ee}} \mathbf{e}_{i}+\sum_{j}\left[\overline{\mathbf{F}}_{i j}^{\mathrm{eh}} \mathbf{h}_{j}-\overline{\mathbf{F}}_{i j}^{\mathrm{ee}} \mathbf{e}_{j}\right]-\mathbf{t}_{i}^{\mathrm{ee}}+\mathbf{t}_{i}^{\mathrm{eh}}-\mathbf{b}_{i}^{\mathrm{ee}}+\mathbf{b}_{i}^{\mathrm{eh}} \\
\mu_{i} \overline{\mathbf{M}}_{i}^{\mathrm{h}} \partial_{t} \mathbf{h}_{i} & =\left[-\overline{\mathbf{S}}_{i}^{\mathrm{he}}+\overline{\mathbf{F}}_{i i}^{\mathrm{he}}\right] \mathbf{e}_{i}+\overline{\mathbf{F}}_{i i}^{\mathrm{hh}} \mathbf{h}_{i}-\sum_{j}\left[\overline{\mathbf{F}}_{i j}^{\mathrm{he}} \mathbf{e}_{j}+\overline{\mathbf{F}}_{i j}^{\mathrm{hh}} \mathbf{h}_{j}\right]-\mathbf{t}_{i}^{\mathrm{hh}}-\mathbf{t}_{i}^{\mathrm{he}}-\mathbf{b}_{i}^{\mathrm{hh}}-\mathbf{b}_{i}^{\mathrm{he}}
\end{aligned}
$$

$\mathbf{e}_{i}$ and $\mathbf{h}_{i}$ are vectors that store the unknown coefficients $f_{i, k}^{\mathrm{e}}$ and $f_{i, h}^{\mathrm{h}}$, respectively. $\overline{\mathbf{M}}, \overline{\mathbf{S}}$, and $\overline{\mathbf{F}}$ are matrices from DGTD. $\mathbf{t}$ and $\mathbf{b}$ are vectors related to incident fields and TDBI, respectively.

\section{NUMERICAL RESULTS}

The excitation is a plane wave propagating a modulated Gaussian pulse expressed:

$$
\mathbf{E}^{i}(\mathbf{r}, t)=\hat{\mathbf{x}} G(t-\mathbf{r} \cdot \hat{\mathbf{z}} / c) \quad G(t)=e^{-\left(t-t_{0}\right)^{2} / \tau_{m}^{2}} \cos \left[2 \pi f_{m}\left(t-t_{0}\right)\right]
$$

Example 1: Three perfect electrically conducting (PEC) spheres

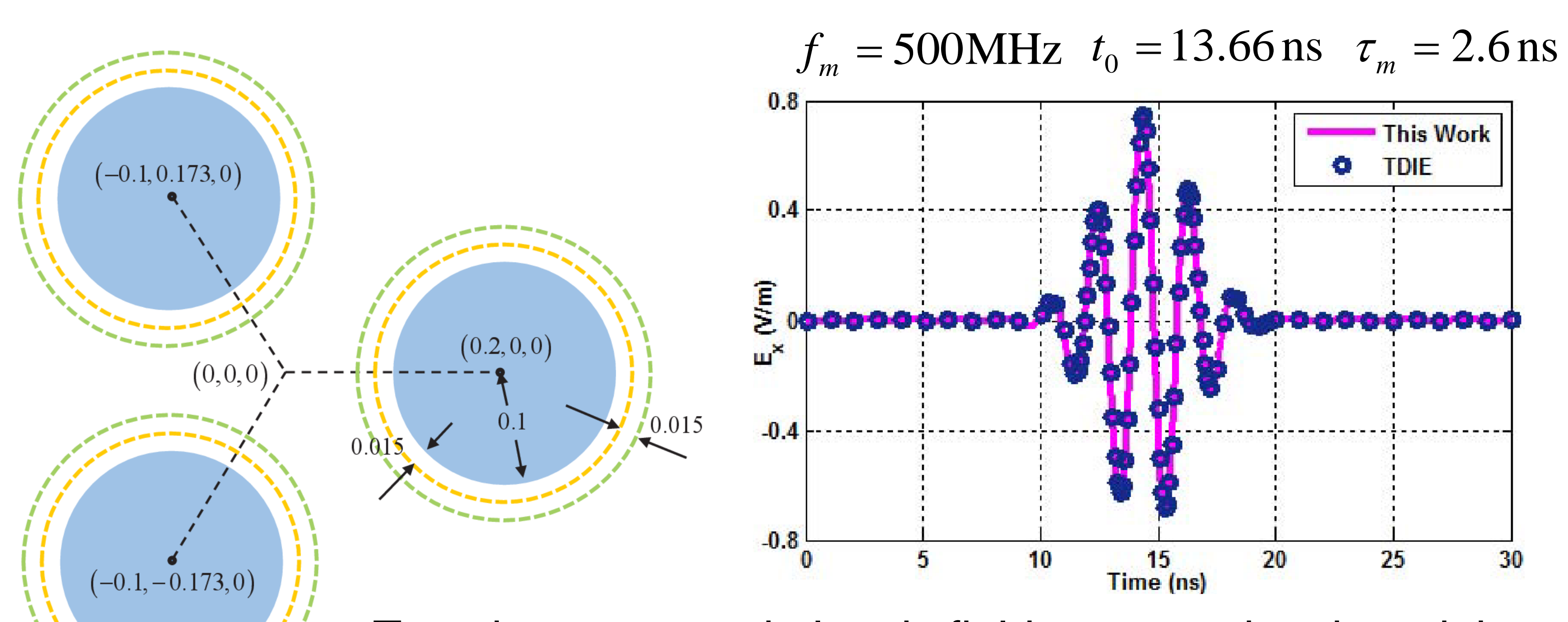

Transient scattered electric field computed at the origin.
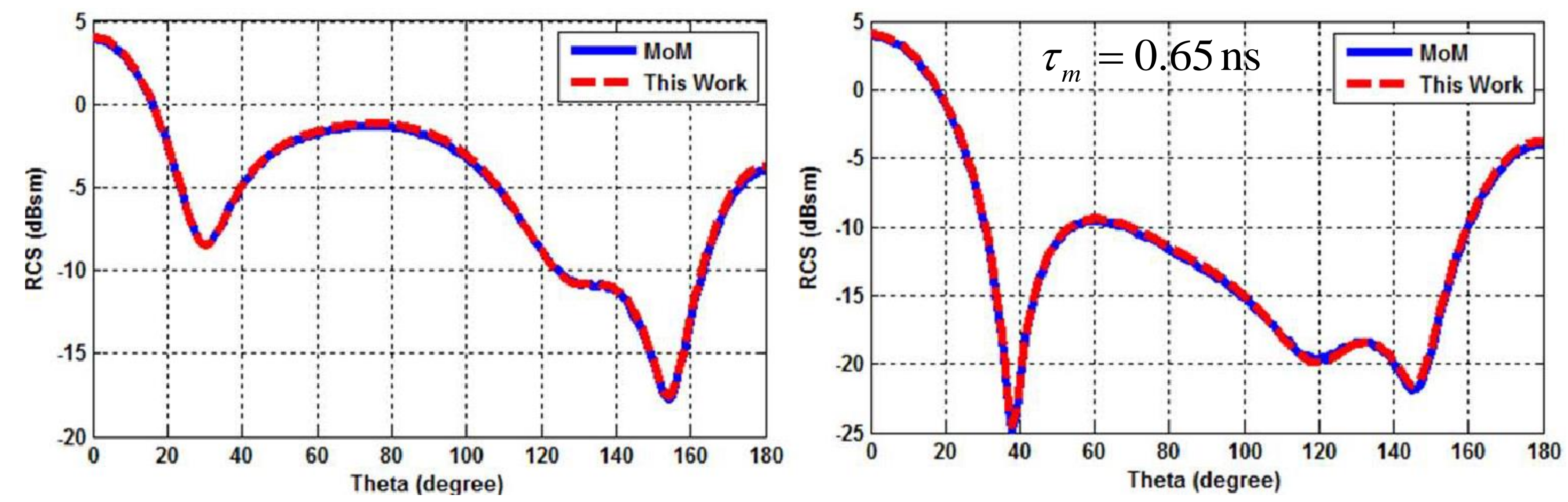

RCS on the $x y$ and $x z$ planes at $2.53 \mathrm{MHz}$, respectively.

Example 2: A dielectric U-shape scatterer

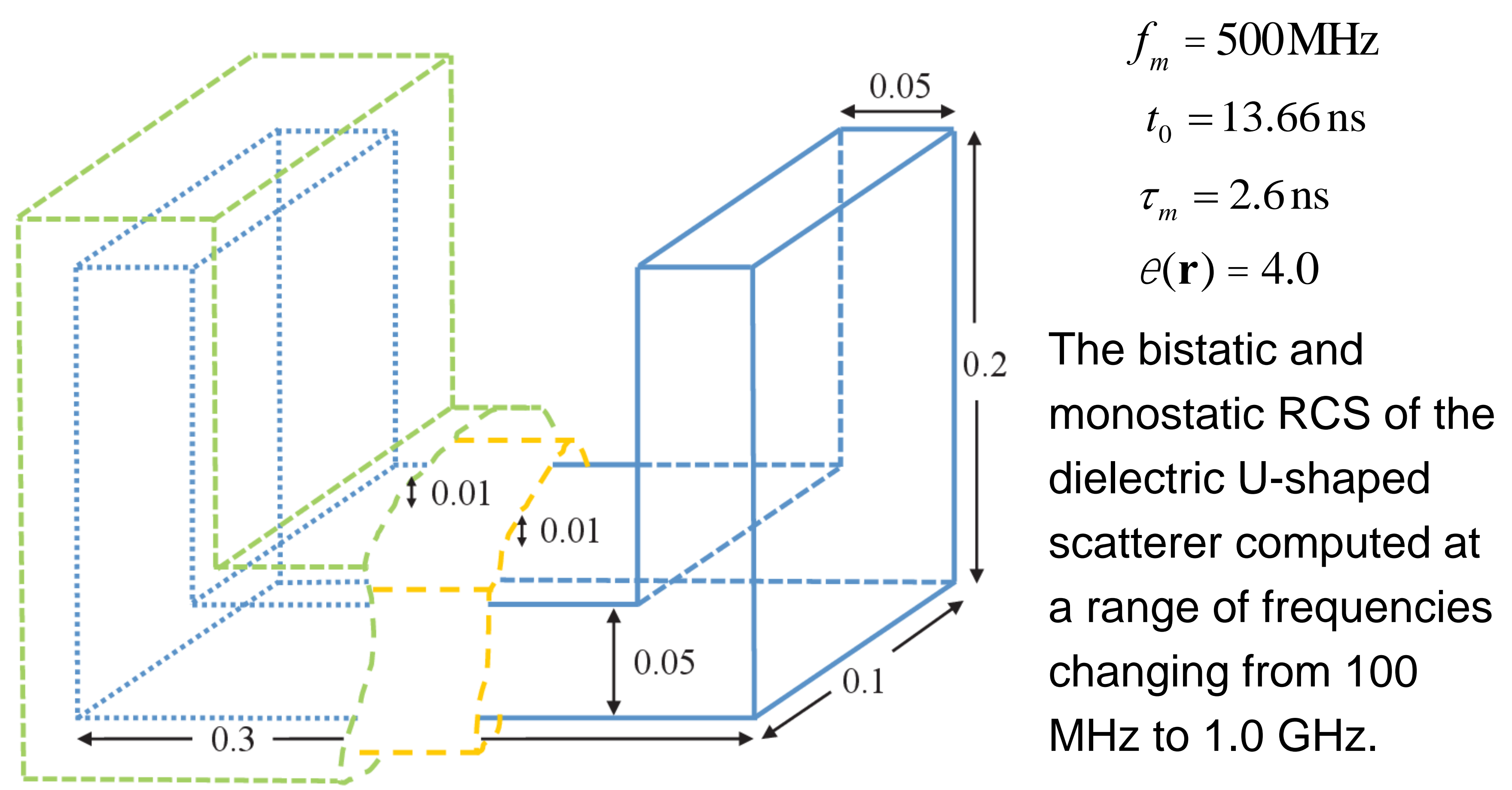

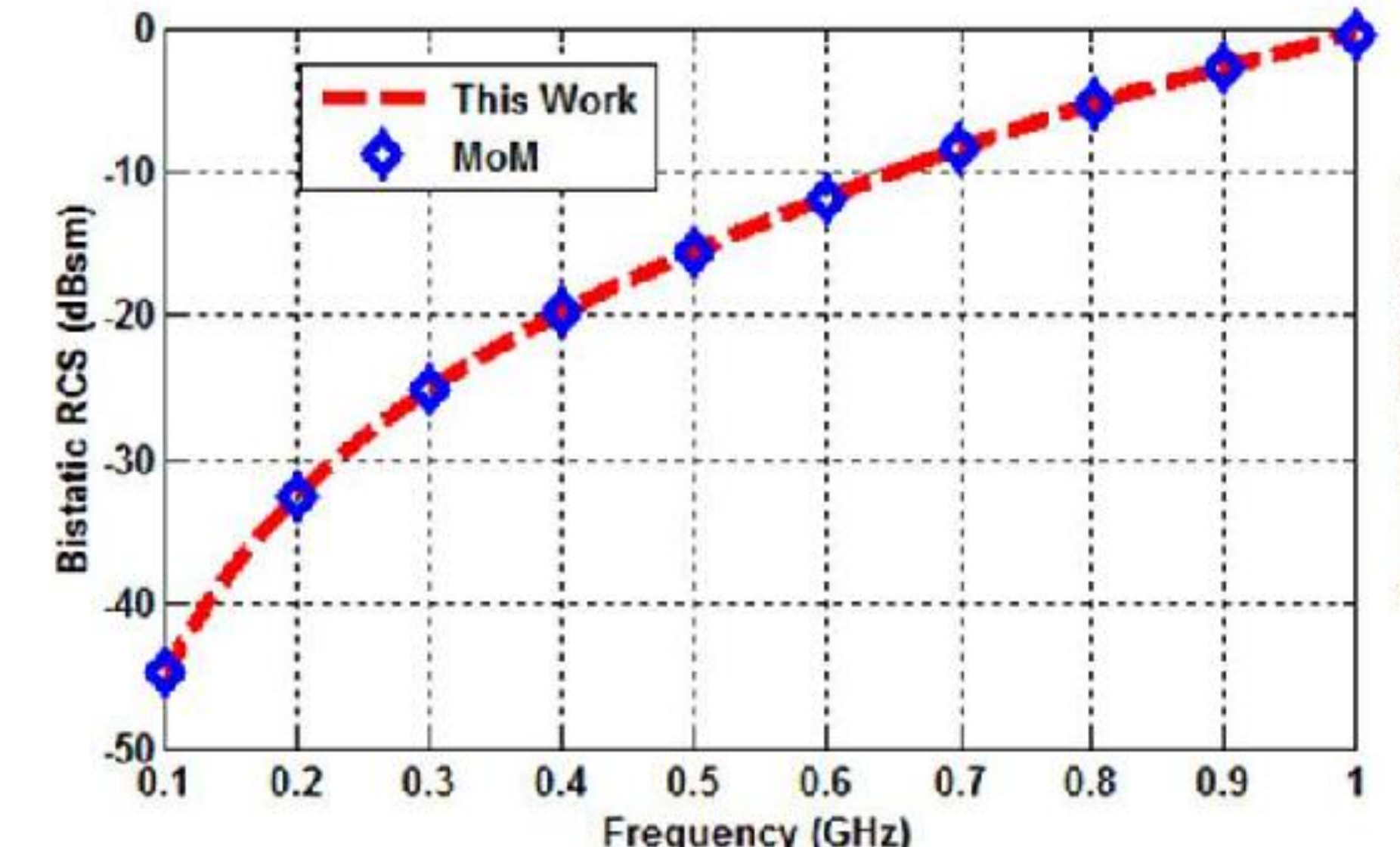

(a)

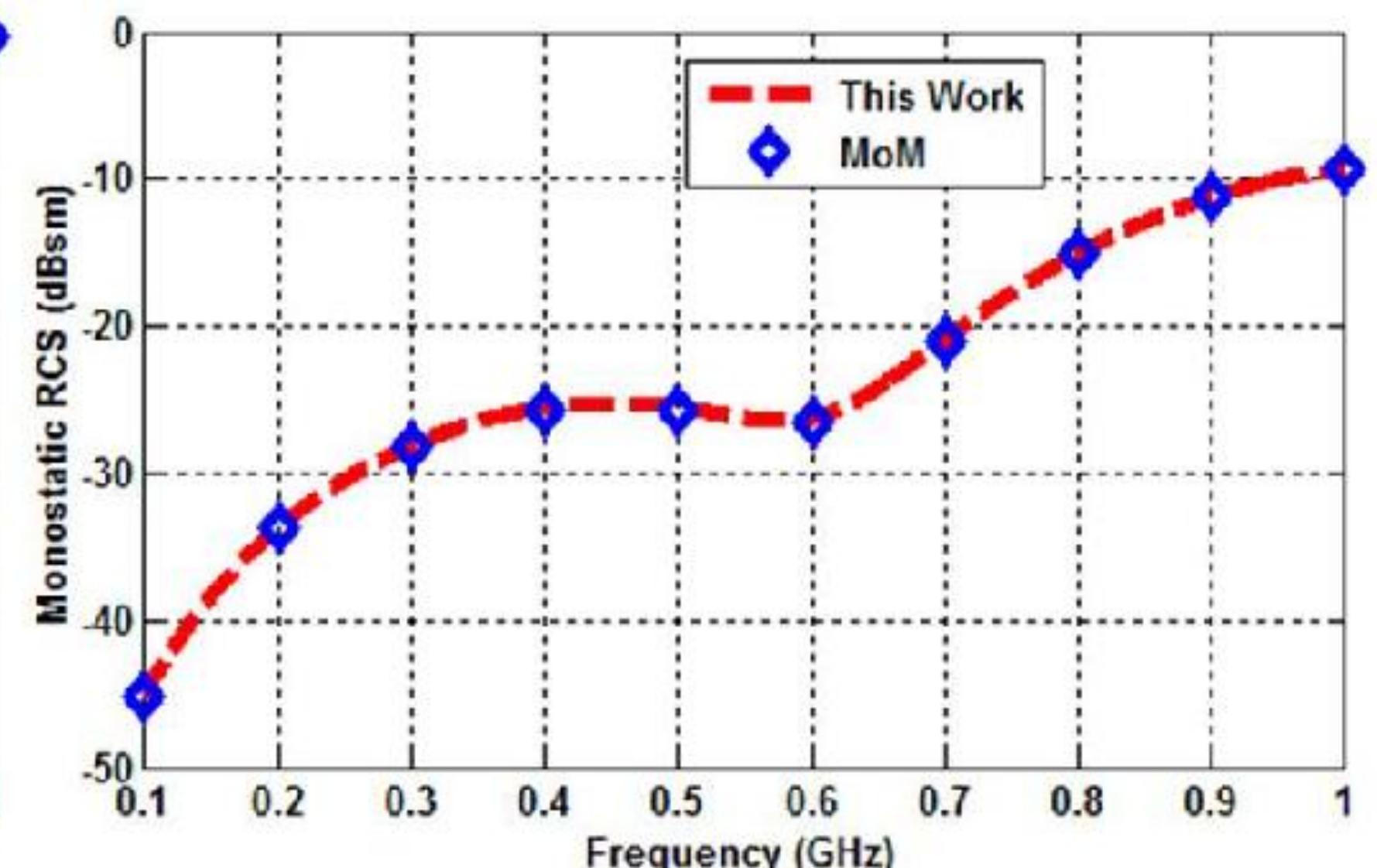

(b)
ADVANTAGES

Disconnected truncation boundaries: Efficient discretization of multiple scatterers. Reduced number of unknowns

Concave truncation boundaries: Efficient discretization of complex geometries. Reduced number of unknowns

Accurate at low-frequencies

Explicitness of DGTD is maintained for efficiency

\section{References}

[1] S. D. Gedney, C. Luo, J. A. Roden, R. D. Crawford, B. Guernsey, J. A. Miller, T. Kramer, and E. W. Lucas, "The discontinuous Galerkin finite element time-domain method solution of Maxwell's equations," ACES J., vol. 24, no. 2, pp. 129-142, Apr. 2009.

[2] D. Jiao, A. A. Ergin, B. Shanker, E. Michielssen, and J. M. Jin, "A fast higher-order time-domain finite element-boundr

for 3 . 